\title{
Проявление РТ-симметрии в экситонных спектрах квантовых ям
}

\author{
(C) В.П. Кочерешко ${ }^{1}$, Л.В. Котова ${ }^{1}$, И.С. Хахалин ${ }^{1}$, R.T. Cox $^{2}$, H. Mariette $^{2}$, \\ R. Andre $^{2}$, H. Bukari' ${ }^{2}$, C.В. Иванов ${ }^{1}$ \\ ${ }^{1}$ Физико-технический институт им. А.Ф. Иоффре Российской академии наук, \\ 194021 Санкт-Петербург, Россия \\ ${ }^{2}$ Institut Nél, CNRS/UJF 25, \\ Avenue des Martyrs BP 166, Fr-38042 Grenoble Cedex 9 \\ E-mail: Vladimir.Kochereshko@mail.ioffe.ru
}

(Получена 27 апреля 2017 г. Принята к печати 12 мая 2017 г.)

В спектрах возбуждения фотолюминесценции и спектрах отражения при круговой поляризации падающего света в широких квантовых ямах, ширина которых много больше боровского радиуса экситона, обнаружено, что в отсутствие магнитного поля отношение интенсивностей четных и нечетных пиков поглощения меняется на противоположное при изменении знака круговой поляризации на противоположный.

DOI: 10.21883/FTP.2017.12.45171.34

\section{1. Введение}

Поляритоны, или светоэкситоны, в кристаллах изучаются со времени первых работ Томаса и Хапфилда [1] и С.И. Пекара [2]. Поляритон является по сути дела фотоном в среде и все выводы, полученные для экситонных поляритонов, относятся к области фундаментальной оптики и поэтому важны для общего понимания оптических процессов в кристаллах и наноструктурах [3]. Казалось бы, здесь все уже хорошо изучено и нет причин ждать, что обнаружатся какие-нибудь новые явления. Однако такой вывод преждевременный, и даже в такой классической области оптики все еще можно найти много необычного. Одно из таких необычных явлений и представлено в данной статье. Было обнаружено, что в квантовой яме коэффициент поглощения света в правой круговой поляризации на четных уровнях квантования экситона больше коэффициента поглощения в левой круговой поляризации, а на нечетных уровнях в точности наоборот, коэффициент поглощения в правой поляризации меньше коэффициента поглощения в левой круговой поляризации. Это выглядит как проявление одной из фундаментальнейших симметрий в природе $P T$-симметрии (P - оператор четности, $T-$ операция инверсии времени). Впервые проявление $P T$-симметрии в спектрах атомов сформулировано в правиле Лапорта: „Энергетические уровни сложных атомов могут быть четными и нечетными. Испускание фотона ведет к переходам, при которых нечетный уровень переходит в четный“.

\section{2. Эксперимент}

Исследовались структуры с широкими квантовыми ямами CdTe/CdZnTe, ZnTe/ZnMgTe и GaAs/AlGaAs, шириной от 50 до 200 нм. В спектрах отражения и возбуждения фотолюминесценции (PLE) при отсутствии поляризации падающего света в таких структурах наблюдались серии линий, соответствующих квантованию движения центра масс экситона. В симметричной квантовой яме уровни квантования экситона классифицируются как четные, $\propto \cos K_{N} z$, или нечетные, $\propto \sin K_{N} z$, в соответствии с четностью волновой функции по отношению к операции отражения $(z \rightarrow-z)$ относительно центра ямы.

В зависимости от ширины ямы в спектрах можно наблюдать либо преимущественно четные уровни квантования при $L=\lambda / 2 n$, либо нечетные уровни при $L=\lambda / n$, либо четные и нечетные вместе, когда $L=3 \lambda / 2 n(L-$ ширина ямы, $\lambda$ - длина волны света, $n-$ показатель преломления света) [4]. Эта зависимость спектра от ширины ямы связана с преимущественным перекрытием волновых функций экситона на четных или нечетных уровнях квантования с полем электромагнитной волны [5]:

$$
\int_{-L / 2}^{L / 2} \Phi(z) E(z) d z .
$$

Здесь $E(z)$ - электромагнитное поле, $E(z)=E e^{i k z-i \omega t}$ $=E e^{-i \omega t}(\cos k z+i \sin k z), \Phi(z)-$ волновая функция экситона.

Поскольку интервал интегрирования симметричен в симметричной яме, то для четных уровней квантования экситона перекрытие не равно нулю только с четными компонентами электромагнитного поля, $\propto \cos k z$, а для нечетных уровней - только с нечетными, $\propto \sin k z$.

На рис. 1, a показан спектр отражения, снятый от структуры с квантовой ямой $\mathrm{CdTe} / \mathrm{Cd}_{0.7} \mathrm{Mg}_{0.3}$ Те шири-

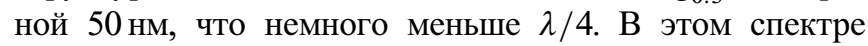
можно видеть, что четные уровни квантования проявляются в спектре заметно ярче, чем нечетные уровни. Это связано с тем, что яма слишком узкая и амплитуда нечетных компонент электромагнитного поля $\propto \sin k z$ 

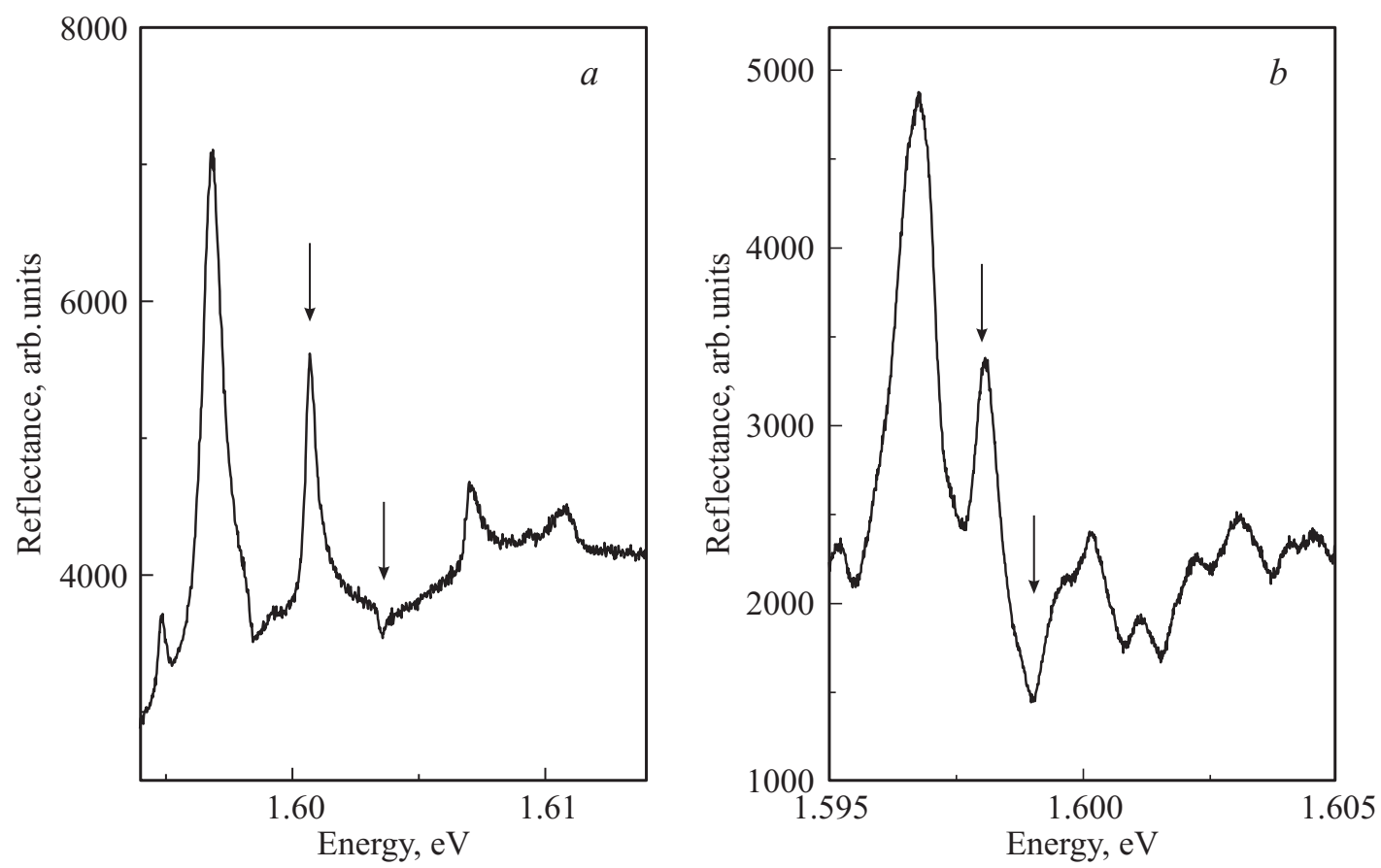

Рис. 1. Спектры отражения от структуры CdTe/CdMgTe с квантовой ямой шириной $50(a)$ и 120 нм $(b)$ при нормальном падении неполяризованного света. Температура измерений $10 \mathrm{~K}$.

слишком мала, а следовательно, мал и интеграл перекрытия (1).

На рис. $1, b$ представлен спектр отражения структуры с квантовой ямой также на основе CdTe, но большей ширины, равной $\sim 120 \mathrm{Hм}$, что несколько больше $\lambda / 2$. В этом спектре перекрытие нечетных состояний с электромагнитным полем заметно больше и интенсивность нечетных линий сравнима с интенсивностью четных.

Удивительное явление было обнаружено в этих структурах в спектрах возбуждения фотолюминесценции (PLE) при циркулярной поляризации падающего света. На рис. 2, $a$ представлен спектр PLE, снятый от структуры CdTe/CdZnTe с шириной ямы $\sim 100$ нм при циркулярно поляризованном возбуждении в правой $\left(\sigma^{+}\right)$и левой $\left(\sigma^{-}\right)$круговых поляризациях и неполяризованном детектировании в нулевом магнитном поле. В условиях

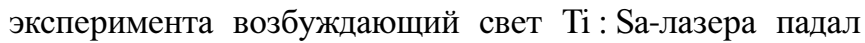
на образец под небольшим углом, $\sim 10^{\circ}$. Фотолюминесценция собиралась в угле $\sim(25-30)^{\circ}$. Сигнал детектировался на длинноволновом крае самой нижней по энергии экситонной линии, соответствующем различным локализованным состояниям. В нашей установке мы не могли использовать нормальное падение света, но считали, что небольшой угол падения не скажется принципиально на полученных спектрах, так как внутри кристалла этот угол еще меньше. Из рисунка хорошо видно, что в правой круговой поляризации в спектре PLE интенсивность нечетных линий заметно превышает интенсивность четных, а при левой круговой поляризации, напротив, интенсивность четных линий превосходит интенсивность нечетных.
Вообще говоря, спектр PLE должен быть эквивалентен спектру поглощения. Следовательно, эксперимент показывает, что спектр поглощения зависит от состояния циркулярной поляризации падающего света. На нечетных уровнях квантования экситона поглощение в правой круговой поляризации больше, чем в левой, a на четных уровнях в левой круговой поляризации больше, чем в правой. Если сложить эти два спектра, то в сумме получаем спектр такой же, как и при неполяризованном возбуждении (рис. 2,b). Получается, что имеет место эффект, похожий на эффект комбинированной $P T$-симметрии, когда при инверсии углового момента спектр PLE изменяется по-разному для четных и нечетных состояний, но при одновременном изменении четности и углового момента спектр сохраняется.

Это, на первый взгляд, выглядит удивительно и указывает на проявление неэквивалентности правого и левого в оптике.

Конечно, в спектрах PLE мы не можем разделить процесс поглощения и процесс релаксации возбуждения, однако от этого наблюдаемый эффект не становится менее удивительным. В спектрах PLE могут проявиться и процессы спин-зависимой релаксации, хотя это не типично для структур на основе CdTe и ZnTe, так как в них, как правило, не наблюдается сигнала оптической ориентации.

Для того чтобы выяснить причины необычного поведения спектров PLE, мы предприняли исследование поляризации отраженного от структуры света в разных геометриях эксперимента. 

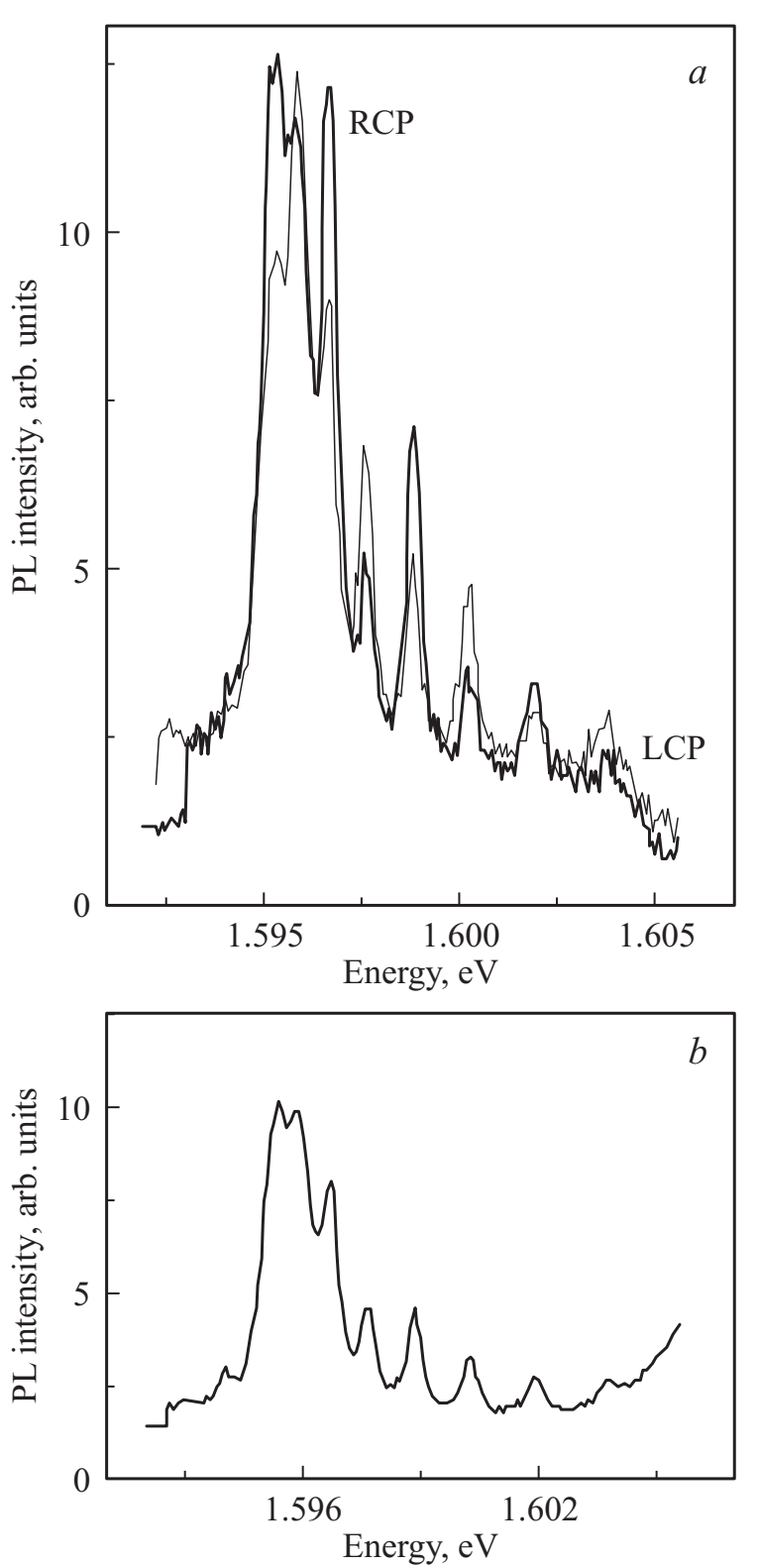

Pис. 2. Спектры возбуждения фотолюминесценции при температуре $10 \mathrm{~K}: a-$ в двух круговых поляризациях $\sigma^{+}(\mathrm{RCP})$ и $\sigma^{-}$(LCP) возбуждающего света в нулевом магнитном поле; $b-$ при неполяризованном возбуждении.

Вообще говоря, конверсия правой круговой поляризации в левую и наоборот в спектрах отражения/пропускания возможна только благодаря двум причинам: явлению гиротропии или явлению двулучепреломления. В кубическом кристалле симметрии $T_{d}$ и в квантовой яме с симметрией $D_{2 d}$ гиротропия отсутствует. Таким образом, наблюдаемый эффект, скорее всего, может быть связан с явлением двулучепреломления. Двулучепреломление может быть вызвано как механическими напряжениями, так и естественными причинами, например пространственной дисперсией [6]. Мы считаем, что механические напряжения в плоскости структуры слишком малы, для того чтобы приводить к такому большому эффекту в поляризации.

Были исследованы спектры отражения при нормальном и наклонном падении при разных поляризациях падающего и отраженного света. Спектры снимались при падении линейно поляризованного света в поляризациях вдоль осей [100], [010] или [110], [11̄0], света круговой поляризации $\sigma^{+}, \sigma^{-}$и при таком же наборе детектируемых поляризаций.

При нормальном падении света мы не обнаружили различий спектров, снятых в разных поляризациях. При наклонном падении под углом $45^{\circ}$, если плоскость линейной поляризации лежала в плоскости $(P$-компонента $)$ или перпендикулярно плоскости ( $S$-компонента) падения, также не наблюдалось различий в характере спектров, снятых в разных поляризациях в разных осях. Хотя общая интенсивность сигнала могла различаться для разных поляризаций, соотношение интенсивностей соседних линий в спектрах сохранялось.

При падении линейно поляризованного света вдоль осей $[100]$ и [010] в наклонной геометрии, когда имелись как $S$-, так и $P$-компонента поляризации, мы также не обнаружили различий при всех детектируемых поляризациях. Сильное различие характера спектров наблюдалось только в наклонной геометрии, когда имелись как $S$-, так и $P$-компонента вдоль осей [110] и [11̄0].

Заметное различие формы спектров проявлялось при наклонном падении циркулярно поляризованного света и детектировании отраженного в циркулярной поляризации. При этом в целом спектры при $\sigma^{+} \rightarrow \sigma^{+}$и $\sigma^{-} \rightarrow \sigma^{-}$совпадали друг с другом (падал и регистрировался сигнал в одинаковых поляризациях) и сильно отличались от спектров в скрещенных поляризациях $\left(\sigma^{+} \rightarrow \sigma^{-}\right.$или $\left.\sigma^{-} \rightarrow \sigma^{+}\right)$. Однако сравнение таких спектров отражения затруднено тем, что их общая интенсивность сильно различалась.

Для того чтобы было удобно сравнивать спектры в разных поляризациях, приведем спектры отражения, снятые в условиях, когда падающий свет был линейно поляризован в наклонной геометрии и имелись как $S$-, так и $P$-компонента вдоль осей $[110]$ и $[1 \overline{1} 0]$ кристалла, a регистрировался свет в круговой поляризации. На рис. 3, $a$ представлены спектры отражения от структуры с квантовой ямой $\mathrm{ZnTe} / \mathrm{Zn}_{0.8} \mathrm{Mg}_{0.1}$ Те шириной 50 нм, когда падающий свет был линейно поляризован вдоль оси [110] образца в наклонной геометрии, а детектировался сигнал в поляризациях $\sigma^{+}$и $\sigma^{-}$. В области низких энергий видны особенности, связанные с квантованием экситона с тяжелой дыркой. Видно, что отношение интенсивностей соседних четных и нечетных линий заметно различается. Отношение интенсивностей четных и нечетных линий в спектре отражения менялось на противоположное при смене знака поляризации.

На рис. $3, b$ представлены спектры отражения структуры на основе $\mathrm{CdTe} / \mathrm{CdZnTe}$, снятый в таких же условиях. Помимо различия отношения амплитуд четных и нечет- 

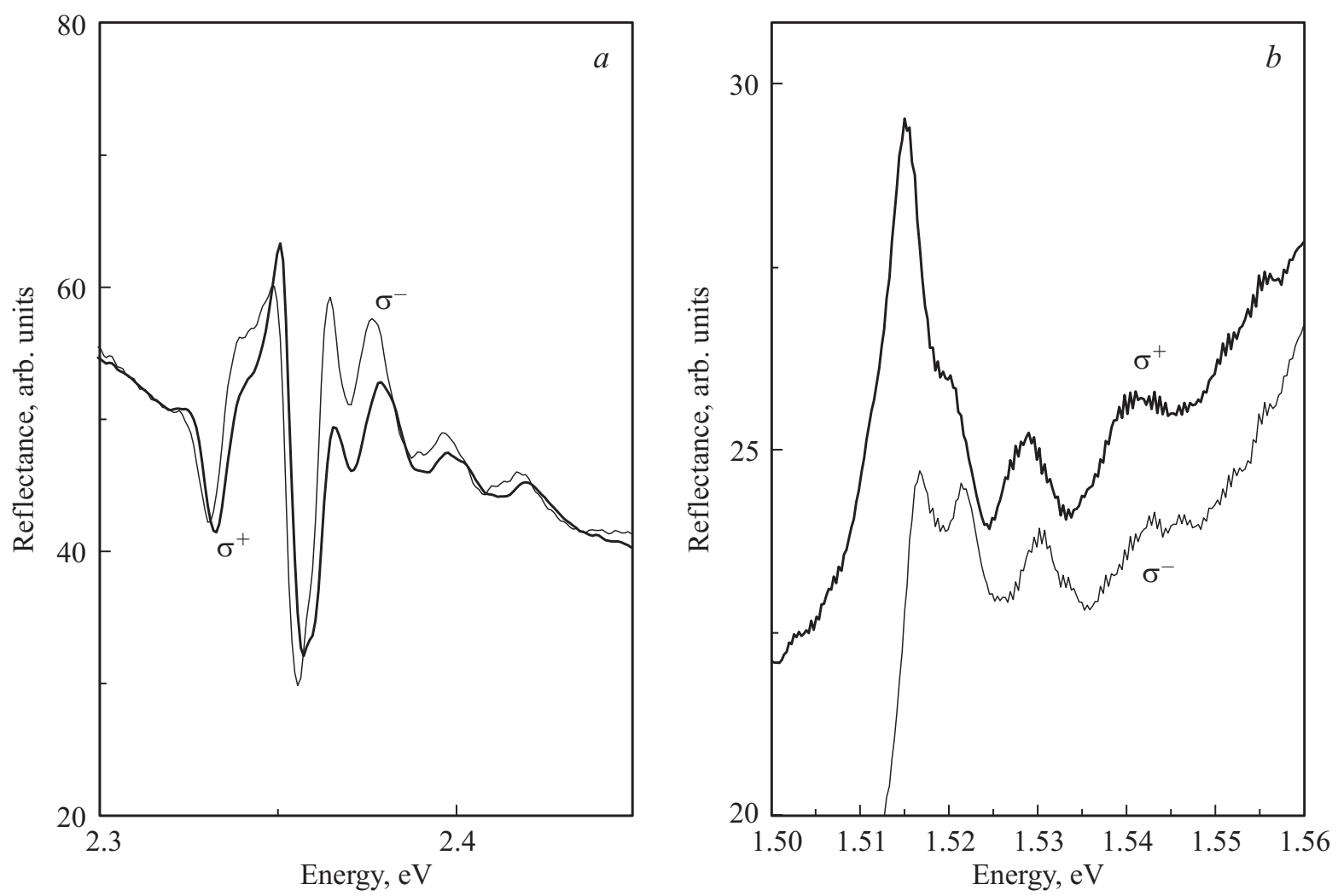

Рис. 3. Спектры отражения от структур с квантовой ямой $\mathrm{ZnTe} / \mathrm{ZnMgTe}$ шириной 50 нм $(a)$ и с квантовой ямой CdTe/CdZnTe шириной 50 нм $(b)$ при $10 \mathrm{~K}$ и наклонном падении под углом $45^{\circ}$. Падающий свет линейно поляризован вдоль оси [110] (наклонная поляризация $45^{\circ}$ по отношению к комплнентам $P$ и $S$ ), регистрировался сигнал в правой $\left(\sigma^{+}\right)$и левой $\left(\sigma^{-}\right)$круговых поляризациях.

ных линий в этом спектре виден и сдвиг всех линий в зависимости от поляризации.

\section{3. Обсуждение}

Поляризационные зависимости однозначно указывают на то, что мы имеем дело с явлением двулучепреломления. Естественной причиной для двулучепреломления может являться пространственная дисперсия. На это указывает и отсутствие эффекта при нормальном падении света, когда латеральные компоненты волнового вектора равны нулю.

В нашем случае эксперимент ставился при наклонном падении под углом $45^{\circ}$, т.е. в геометрии $K_{z} \|[001]$, $K_{\perp} \|$ [110]. Добавка в тензор диэлектрической проницаемости, связанная с квадратичной пространственной дисперсией в объемном кубическом кристалле симметрии $T_{d}$, имеет вид

$\delta \varepsilon_{i j}\left(K^{2}\right)=\left(\begin{array}{ccc}B_{1} K_{\perp}^{2}+B_{2} K_{z}^{2} & 0 & B_{3} K_{z} K_{\perp} \\ 0 & B_{1} K_{\perp}^{2}+B_{2} K_{z}^{2} & 0 \\ B_{3} K_{z} K_{\perp} & 0 & B_{2} K_{\perp}^{2}+B_{1} K_{z}^{2}\end{array}\right)$.

Таким образом, при учете пространственной дисперсии наша структура ведет себя как одноосный кристалл, одна из оптических осей которого совпадает с осью $y$, с направлением [1ㅣㅣ, а вторая ось лежит в плоскости $(x, z)(110)$. Диэлектрические проницаемости вдоль и перпендикулярно оптической оси не совпадают, и именно это приводит к эффекту двулучепреломления. В результате при падении линейно поляризованного вдоль оси [100] света отраженный свет оказывается поляризованным эллиптически.

Эти слагаемые, описывающие пространственную дисперсию в диэлектрическом тензоре, происходят из кубических слагаемых $\left(\gamma_{2}-\gamma_{3}\right) \sum K_{i} K_{j}\left\{J_{i} J_{j}\right\}$ в гамильтониане Латтинжера. Они приводят к смешиванию продольной (вдоль $z$ ) компоненты легкого экситона и поперечных компонент тяжелого экситона. Формула (2) написана для объемного кубического кристалла. Для квантовой ямы $K_{z}$ является градиентом по оси $z: K_{z}=-i \nabla_{z}$.

Благодаря наличию слагаемого с градиентом по $z$ нечетные волновые функции $\propto \sin K_{2 N} z$ примешиваются к четным функциям $\propto \cos K_{2 N} z$, а четные к нечетным:

$$
\begin{gathered}
\varphi_{2 N}(z)=A_{2 N}\left(\cos K_{2 N} z-\beta K_{x} K_{2 N} \sin K_{2 N} z\right), \\
\varphi_{2 N+1}(z)=A_{2 N+1}\left(\sin K_{2 N+1} z+\beta K_{x} K_{2 N+1} \cos K_{2 N+1} z\right) .
\end{gathered}
$$

В результате этого для нечетных состояний квантования экситона к свету, поляризованному по правому 
кругу, будут добавляться компоненты, поляризованные вдоль оси $z$, а для четных состояний будут вычитаться $z$-компоненты поляризации. Для света, поляризованного по левому кругу, ситуация будет в точности обратной.

В спектрах отражения это может наблюдаться только при наклонном падении. Проявление же эффекта $P T$-симметрии в спектрах PLE связано с тем, что при регистрации сигнала ФЛ всегда есть непараллельность пучка. Мы сняли спектры отражения при углах падения $\sim 10^{\circ}$. Оказалось, что и при таких малых углах есть различие спектров в разных поляризациях, хотя и не столь заметное, как при $45^{\circ}$.

\section{4. Заключение}

В спектрах отражения и возбуждения фотолюминесценции при круговой поляризации падающего света при наклонном падении в широких квантовых ямах, ширина которых много больше боровского радиуса экситона, обнаружено, что в отсутствие магнитного поля отношение интенсивностей четных и нечетных пиков поглощения меняется на противоположное при изменении знака круговой поляризации на противоположный.

Эффект объясняется в модели, учитывающей эффект двулучепреломления, вызванного пространственной дисперсией экситона. Сам эффект двулучепреломления проявлялся в конверсии линейной поляризации в циркулярную поляризацию проходящего (отраженного) света и, напротив, из циркулярной в линейную, при этом знак конверсии зависел от четности уровня квантования. В результате при одновременном изменении четности уровня и знака поляризации ничего не менялось.

Работа получила частичную финансовую поддержку в рамках гранта РФФИ № 15-02-04527a.

\section{Список литературы}

[1] J.J. Hopfield. Phys. Rev., 112, 1555 (1958) .

[2] S.I. Pekar. Fiz. USSR, 33, 1022 (1957).

[3] В.М. Агранович, В.Л. Гинзбург. Кристаллооптика с учетом пространственной дисперсии и теория экситонов (М., Наука, 1965).

[4] Y. Merle d'Aubigne, Le Si Dang, A. Wasiela et al. J. Physique C5, 48, 363 (1987).

[5] E.L. Ivchenko. Optical Spectroscopy of Semiconductor Nanostructures (Alpha Science Int., Harrow, UK, 2005).

[6] Е.Ф. Гросс, А.А. Каплянский. ДАН СССР, 132, 98 (1960).

Редактор Л.В. Шаронова

\section{Manifestation of $P T$ symmetry in exciton spectra of quantum wells}

\author{
V.P. Kochereshko ${ }^{1}$, L.V. Kotova ${ }^{1}$, I.S. Khakhalin', \\ R.T. Cox ${ }^{2}$, H. Mariette ${ }^{2}$, R. Andre ${ }^{2}$, H. Bukari ${ }^{2}$, \\ S.V. Ivanov ${ }^{1}$ \\ ${ }^{1}$ loffe Institute, \\ 194021 St. Petersburg, Russia \\ 2 Institut Nél, CNRS/UJF 25, \\ Avenue des Martyrs BP 166, \\ Fr-38042 Grenoble Cedex 9
}

Abstract In the photoluminescence excitation and reflection spectra it was found that at circular polarized incident light in wide quantum wells, with width much larger than the exciton Bohr radius, in the absence of a magnetic fields the ratio of the intensities of even and odd absorption peaks changes to the opposite when the sign of the circular polarization reverses. 